\title{
A case of Takayasu's arteritis and pulmonary artery involvement
}

\section{Kotsonis ${ }^{1}$}

A.Eliades $^{1}$

N.

Chatzigiannis ${ }^{1}$

G. Markou ${ }^{1}$

1.Pediatric

Intensive Care Unit, University

Hospital of

Patras, Greece

\section{Department of} Pediatrics, Childre

$\mathrm{n}$ Hospital of Patras, Greece

\section{Introduction}

Takayasu's arteritis (TA) is a chronic vasculitis of unknown etiology, predominantly affecting aorta and its branches with various signs depending on the area and severity of vascular involvement.

\section{Case description}

An 11-year-old girl was admitted in PICU hemodynamically unstable , hypoxaemic and anouric. Her past medical history reported aortic isthmus stenosis at the age of 6, hypertension, claudication, fatigue and a recent diagnosis of TA. Due to critical condition, she was intubated and stabilised using inotropic and diuretic drugs. Noted that catheterisation was proven difficult due to unpalpable pulse at lower extremities. Her symptoms were attributed to TA. Echo cardiography revealed left ventricular hypertrophy, whilst CT revealed abnormal wall thickening of common carotid, left subclavian and thoracic aorta, and severe stenosis of pulmonary arteries. Lungs presented with ground glass opacity and pleural effusion. The patient underwent emergent abdominal aorta stent implantation in order to restore kidney perfusion and lower extremity circulation. She was placed on continuous venovenous hemofiltration for 2 days and was gradually improved under treatment with methotrexate and corticosteroids. Months later, the patient was readmitted to PICU due to dyspnea. CTs showed new obstruction at abdominal aorta under the stent so a second stent implantation was performed. She also underwent cardiac catheterization which demonstrated severe pulmonary involvement and systolic pulmonary hypertension. Treatment with sildenafil and esoprostenol was started. Cyclophosphamide was added to corticosteroids because of disease severity.

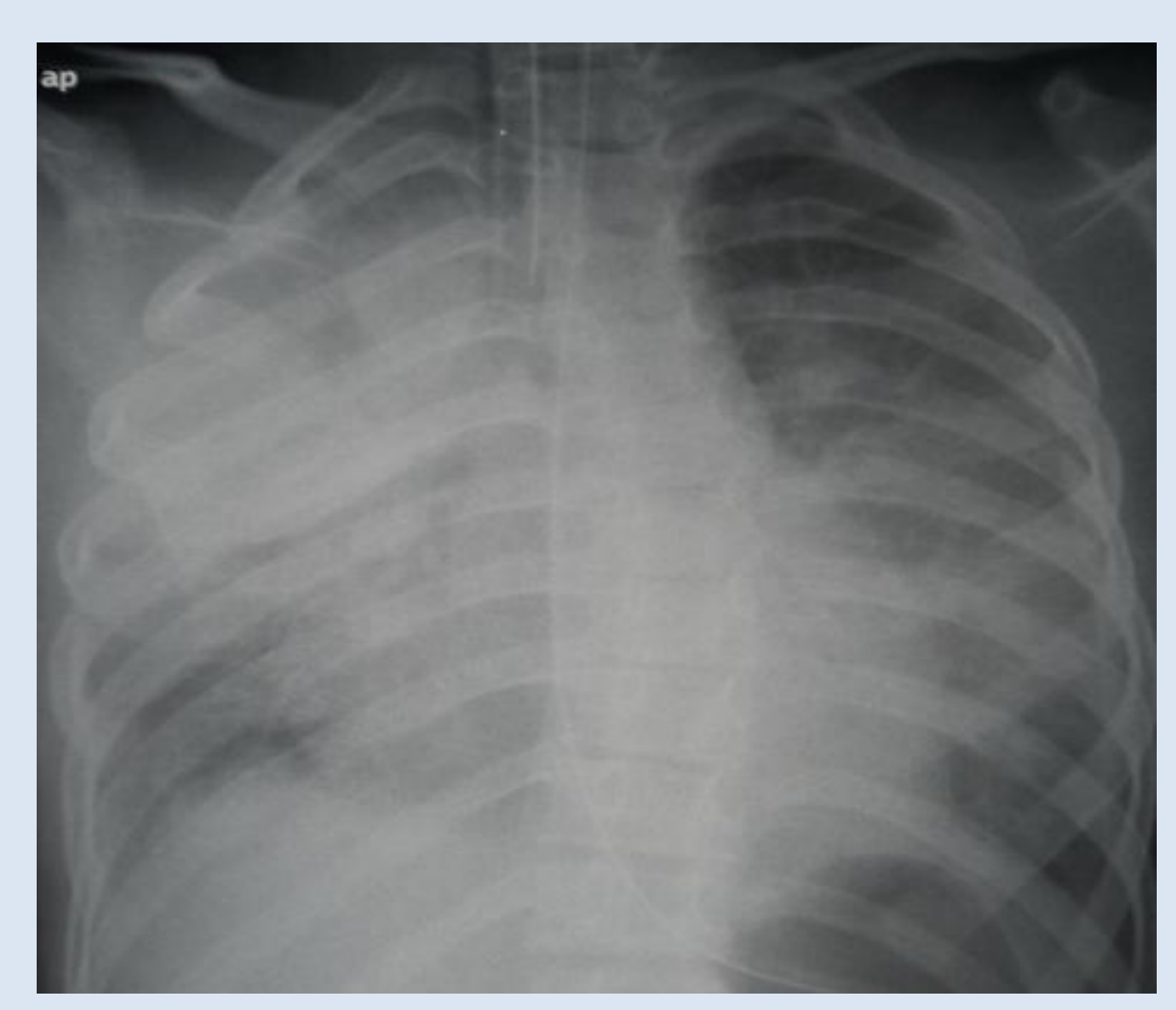

\section{Conclusion}

TA may be complicated by pulmonary arteritis and PAH that should be recognized early and treated promptly for prevention of irreversible vascular damage.

\section{REFERENCES}

1.Nakajima, N., Takayasu arteritis: consideration of pulmonary involvement. Ann Vasc Dis, 2008. 1(1): p. 7-10. 2. Aeschlimann, F.A., et al., Presentation and disease course of childhood- versus adult-onset Takayasu Arteritis. Arthritis Rheumatol, 2018. 